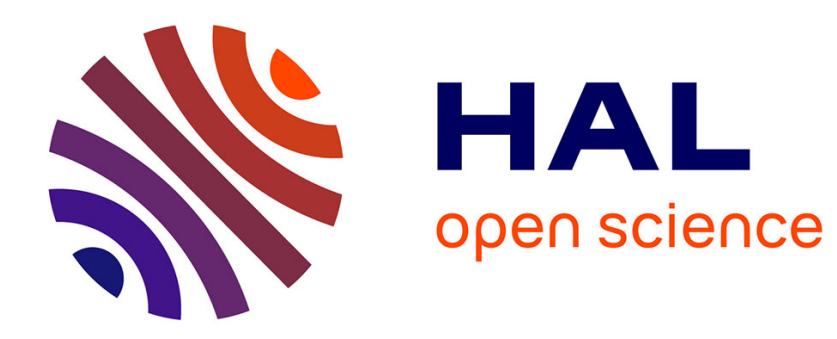

\title{
Ray dispersion strongly modified by a periodic index segmentation
}

\author{
Pierre Aschieri, Valérie Doya
}

\section{To cite this version:}

Pierre Aschieri, Valérie Doya. Ray dispersion strongly modified by a periodic index segmentation.

Optics Communications, 2010, 283 (19), pp.3673. 10.1016/j.optcom.2010.05.026 . hal-00647534

\section{HAL Id: hal-00647534 \\ https://hal.science/hal-00647534}

Submitted on 2 Dec 2011

HAL is a multi-disciplinary open access archive for the deposit and dissemination of scientific research documents, whether they are published or not. The documents may come from teaching and research institutions in France or abroad, or from public or private research centers.
L'archive ouverte pluridisciplinaire HAL, est destinée au dépôt et à la diffusion de documents scientifiques de niveau recherche, publiés ou non, émanant des établissements d'enseignement et de recherche français ou étrangers, des laboratoires publics ou privés. 


\title{
Ray dispersion strongly modified by a periodic index segmentation
}

\author{
Pierre Aschiéri, Valérie Doya \\ Université de Nice-Sophia Antipolis \\ Laboratoire de Physique de la Matière Condensée, UMR 6622 \\ Parc Valrose, Nice, France
}

\begin{abstract}
The purpose of this article is to analyze the impact of an index segmentation on the ray dispersion in the geometrical approach. The presented study reveals that a periodic index segmentation of the waveguide along with a transverse gaussian index variation exhibits complex ray dynamics that strongly modify ray dispersion properties.
\end{abstract}

Key words: Segmented waveguides, Ray dispersion, Chaos, Integrated optics

PACS: 42.82.Et, 42.15.Dp, 05.45.Gg

\section{Introduction}

A Periodic Segmented Waveguide (PSW) is characterized by an array of high index region along the direction of propagation and by a transverse index profile of high index segments which depends on the waveguide fabrication process. Those waveguides are well known to be very interesting for many purpose such as mode tapers or mode filters [1-4], they have also been used to achieve efficient nonlinear guided wave interaction using the 
Quasi Phase Matching (QPM) or Balanced Phase Matching (BPM) schemes [5-7] that take advantage of a periodic reversal of the nonlinear coefficient associated with such waveguides. More recently, it has been shown that multimode PSW can be a practical support for classical chaos investigation in the geometrical limit [8]. The ray dynamics in the simple and ideal 2D case of a PSW characterized by a transverse gaussian index profile is responsible for the emergence of a genuine chaotic behavior of the ray dynamics which is much more complex than that encountered with the usually considered parabolic index profile [9-11]. Such a complex behavior is actually comparable to what has already been studied in a periodically perturbed waveguide $[12,13]$ or more generally, the analysis shows a dynamic that can be found in usual periodically forced nonlinear pendulum. PSW ray dynamics exhibit resonances, frequency locking, as well as a chaotic behavior and as a consequence of this complex aspect of the system, the transit time of rays (e.g. the ray dispersion) in the waveguide is expected to be different as in non segmented waveguide. As observed in previous work [14], it is shown, here, that the dispersion is determined by the topology of the phase space.

After a description of the system used, numerical results of ray dispersion value for two waveguides configurations will then be discussed and finally, conclusions and perspectives will be drawn.

\section{Dispersion calculation}

A schematic representation of a PSW is sketched in figure 1. The PSW presents a transverse gaussian index profile in the high index segments that is, for example, naturally encountered with the proton exchange technique 
waveguide fabrication on $\mathrm{LiNbO}_{3}$ substrate [16]. The duty-cycle of a PSW is usually define as $D C=d / \Lambda$ and a step index profile is assumed along the propagation direction $z$ as it has been done in previous works [8-10]. It can be mentioned that a smoother profile will not change qualitatively the results. Assuming that light wavelength is very small compared to the dimensions of a highly multimode waveguide, ray optics approximation can be used. A ray is define as the path along which light energy is transmitted from one point to another in the optical system with a velocity equal to $c / n(x)$ where $c$ is the speed of light in the vacuum and $n(x)$ is the transverse index profile. In low index segments, the index profile is constant and $n(x)=n_{2}$ whereas the index profile of the high index segments is given by :

$$
n(x)=n_{2}+\delta n e^{-\frac{x^{2}}{w^{2}}}
$$

where $w$ is the width of the waveguide, $\delta n=n_{1}-n_{2}, n_{2}$ is the substrate index and $n_{1}$ is the maximum index induced by the waveguide fabrication process. We consider here dielectric waveguides characterized by a low index contrast $\delta n \ll 1$, which allows us to neglect the reflections of rays at each interfaces.

A ray path in a $2 \mathrm{D}$ medium can be described by the following equation in cartesian coordinates [15]:

$$
\frac{d^{2} x}{d z^{2}}=\frac{1}{2 \beta^{2}} \frac{d n^{2}(x)}{d x}
$$

where, $x$ and $z$ are the transversal and longitudinal coordinates respectively, $n(x)$ is the transverse index profile, $\beta=n\left(x_{i}\right) \cos \theta_{i}$ is the invariant of the ray path, $x_{i}$ being the initial position of the ray and $\theta_{i}$ being the incident angle of the ray respect to $z$ axis. The ray path can be calculated by analyzing high 
and low index segments separately and using the law of refraction at each interface. In the low index segment, the solution is trivial and corresponds to a straight line. In the high index segment characterized by a transverse gaussian index profile, the ray equation (2) is now :

$$
\frac{d^{2} x}{d z^{2}}=-\frac{2 n_{2} \delta n}{\beta^{2} w^{2}} x e^{-\frac{x^{2}}{w^{2}}}
$$

in the limit of usual classical assumption $\delta n \ll 1$. Ray paths of a PSW with a gaussian index profile is plotted on figure 2 for two different initial conditions $\left(\theta_{i}=0^{\circ}, x_{i}=1.7 \mu \mathrm{m}\right)$ and $\left(\theta_{i}=0^{\circ}, x_{i}=0\right)$. When $\theta_{i} \neq 0^{\circ}$ or $x_{i} \neq 0$, ray trajectory oscillate with a period of oscillation $Z_{p}$ whereas the trajectory is a straight line when $\theta_{i}=0^{\circ}$ and $x_{i}=0$ [17]. It is important to note that the gaussian shape profile makes equation (3) nonlinear with respect to variable $x$, whereas, for example, for a parabolic index profile, equation (3) is reduced to a linear equation [8]. For a parabolic PSW, the presence of a linear term in the RHS of equation (3) makes the system analogous to a parametric system [18]. This kind of system can exhibits parametric resonances which are responsible for a ray divergence whatever may be the initial conditions, a feature that has already been study in a previous work [9]. For a gaussian index PSW, the situation is different because the nonlinear term in the RHS of equation (3) saturates the parametric instability and leads to nonlinear resonances which occur when the segmentation period $\Lambda$ and the period of ray oscillation $Z_{p}$ are in a rational ratio [8]. The presence of nonlinear resonances makes the ray dispersion behavior more interesting and some results will be shown in the next sections.

Ray dispersion, also often call intermodal dispersion, is caused by the fact that different rays take different times to propagate along different paths from 
the input to the output of the waveguide. For our purpose, we define the ray dispersion in time $\Delta t$ in $p s / \mathrm{km}$ as the transit time difference between optical path of the considering ray and the straight ray propagating in the center of the waveguide (see figure 2). $\Delta t$ is defined by :

$$
\Delta t=\frac{1}{c}\left(n_{1} L-\int n(x) d s\right)
$$

where $L$ is the length of the waveguide and $d s \simeq \sqrt{d x^{2}+d z^{2}}$ is an arc length along the ray path. In order to get an deeper insight of the ray dynamics and dispersion properties, Poincaré sections were used. Poincaré section consists on a projection at each period of the ray trajectory on to the phase plane $(x, \theta)$ where $x$ is the transverse position of the ray and $\theta$ is the angle that makes the ray with the propagation axis. Along with the Poincaré section, a dispersion map were used where $\Delta t$ values are reported on a graph for different initial conditions except for diverging rays (e. g. rays that escape from the waveguide, $\Delta t \rightarrow \infty)$. Two different transverse index profiles are considered here, a symmetric and a non symmetric one.

\subsection{Symmetric $P S W$}

The figure 3(a) represents the Poincaré section for initial conditions taken in the range of $[-3 \mu \mathrm{m},+3 \mu \mathrm{m}]$ for $x_{i}$ and in the range of $\left[-4^{\circ},+4^{\circ}\right]$ for $\theta_{i}$. Poincaré sections reveal the mixed aspect of the system characterized by the coexistence of a regular or a chaotic behavior of the ray trajectory depending on initial condition. Non divergent rays are characterized by a concentric close circle present on the center of the Poincaré section and also around 4 fixed points, these 4 fixed points are resonances of the system. Resonances occur when $Z_{p}$ and $\Lambda$ are comensurable which is the case here where $Z_{p} / \Lambda \simeq$ 
4. Divergent rays are represented by dots which are mainly present on the peripheral part of the graph. The figure 3(b) represents the corresponding dispersion map for the same PSW, where $\Delta t$ values are represented in a grey level. Uniform grey level regions represent equivalent transit time for trajectories. As it has been mentioned previously, $\Delta t$ are not reported on the graph for diverging rays trajectories in order to keep a clearer picture. These two graphs are similar, the 4 resonances of the Poincaré section are still visible on the dispersion map where 4 wide uniform grey regions are present at the same location. It means that the transit time is similar for rays that have been launched on these resonances. It has to be mentioned that this correspondence between Poincaré section and dispersion map appears for any kind of waveguide configuration. So, a complex Poincaré section structure will lead to a complex dispersion map and then to a non classical dispersion behavior which has been pointed out in previous work [14]. The figure 4 is a slice at $\theta=0^{\circ}$ of the dispersion map for the same waveguide and for the corresponding equivalent continuous waveguide. A PSW can be represented by a continuous equivalent waveguide with an index $n_{e q}(x)=n_{2}+\delta n(x) \times D C$ $[9,10,19]$. The incident angle is fixed and initials conditions are taken such as only the position of the incident ray varies. For the equivalent continuous waveguide, the absolute value of the dispersion increase monotonically as the position of the incident ray moves away from the center of the waveguide. This behavior is not the same with the PSW where the dispersion curve is not monotonic. For stable trajectories, $|x| \leq 0.5 \mu \mathrm{m}$, characterized by a close circle curve on the Poincaré section, dispersion value are approximatively the same as the one of the continuous equivalent waveguide. For $|x|>0.5 \mu \mathrm{m}$, 
the dispersion curve exhibits plateau with a an apex on the edge. The width of the plateau corresponds to the width of the resonance on the Poincaré section. Away from the resonance, the dispersion curve of the PSW goes back to the dispersion curve of the equivalent continuous waveguide.

The ray dispersion behavior is related to the presence of resonances. When $Z_{p}$ and $\Lambda$ are not commensurable, no resonance occurs and ray dispersion is comparable with that of a classical continuous waveguide. Resonances occur when $Z_{p}$ and $\Lambda$ are commensurable and then, as long as the ratio $Z_{p} / \Lambda$ is constant, rays trajectories are synchronized to the segmentation period (this phenomenon is also call frequency locking). The ray is catch by the resonance and a slight modification of the guiding condition does not change ray path and, therefore, the transit time remains almost constant. The range where the initial conditions can be taken without modifying the value of the ray dispersion is determined by the width of the resonance. A strong resonance represented by large islands in the Poincaré section permits a quite large variation of angle or position for the initial conditions without changing $\Delta t$ value.

Singularities in the ray dispersion can be clearly identified with the help of the Poincaré section which is fixed for a given waveguide configuration, but they can occur for a given input condition and changing the waveguide parameters. The figure 5 represents ray dispersion values for different segmentation periods $\Lambda$ as a function of the index difference $\delta n$. Dispersion behavior of a PSW deviate from that of the continuous equivalent waveguide model. Ray dispersion is not monotonic as a function of $\delta n$ and $\Delta t$ can decrease whereas $\delta n$ increase which is not intuitive. 


\subsection{Asymmetric PSW}

The introduction of an asymmetric index profile in the transverse direction (figure 6) emphasizes the non classical dispersion behavior. It has to be said that a non symmetrical as well as a symmetrical gaussian index profile can be technologically achieved. Figure 7(a) represents the Poincaré section of a waveguide with an asymmetrical gaussian index profile and shows a more complex topology regarding the previous one (figure 3). The corresponding dispersion map is represented in figure 7(b) and it is similar to the Poincaré section. Figure 8 represents 3 different slices for $x=0, x=1$ and $x=1.5 \mu \mathrm{m}$ of the dispersion map(figure $7(\mathrm{~b}))$. The presence of resonances show themselves through appearance of plateaus as in the symmetrical waveguide profile. Stochastic ray dispersion values can be seen between plateaus or on the edge of the curve. These random dispersion values are due to the fact that resonances lies in a stochastic sea which can clearly be seen with the Poincaré section (figure 7 (a)). Like in the symmetric waveguide, the dispersion value is frozen if initial conditions are taken in a resonance on the Poincaré section but chaotic elsewhere. Figure 9 represents ray dispersion values for two different segmentation periods $\Lambda=5 \mu \mathrm{m}$ and $\Lambda=10 \mu \mathrm{m}$ as a function of the index difference $\delta n$ for a given input condition. Dispersion values seem to be randomly distributed around a mean value and the deviation from the continuous equivalent waveguide model is then much important.

\section{Conclusions and perspectives}

A simple ray propagation model has been used to provide a numerical study of the dispersion property of a PSW characterized by a gaussian in- 
dex segment profile. The analysis has shown a non standard behavior of the ray dispersion which is related to the complex topology of the Poincaré section and that can not be found in usual waveguides. The deviation from a classical ray dispersion behavior is enhanced with the introduction of an asymmetric transverse index profile. Beside the academic interest that represent a non standard ray dispersion behavior in a multimodes PSW, it might be interesting to consider devices with a periodic index segmentation and with a transverse gaussian index variation. According to the analysis presented here, these devices may exhibit resonances which could help to reduce pulse dispersion in highly multimode communication components for example. However, deeper analysis has to be performed in order to fully investigate the potential of those waveguide, in particular the extension of the analysis to the optical wave domain has to be done. From a technological point of view, no major difficulty seems to exist to achieve an experimental study of our theoretical predictions considering the fact that the waveguide configuration proposed here is based on well known and widely used fabrication process. 


\section{References}

[1] Z. Weissman, A. Hardy, "Modes of periodically segmented waveguides", J. Lightwave Technol., vol. 11, 1993, 1831-1838.

[2] M.H. Chou, M.A. Arbore, and M.M. Fejer, "Adiabatically tapered periodic segmentation of channel waveguides for mode-size transformation and fundamental mode excitation", Opt. Lett., vol. 17, 1996, 794-796.

[3] D. Ortega, J. M. Aldariz, J. M. Arnold, J. S. Aitchison, "Analysis of "Quasi Modes" in Periodic Segmented Waveguides", J. Lightwave Technol., Vol. 17, 1999, 369-375.

[4] Davide Castaldini, Paolo Bassi, Sorin Tascu, Pierre Aschieri, Marc P. De Micheli, and Pascal Baldi, "Soft-Proton-Exchange Tapers for Low Insertion-Loss LiNbO3 Devices", J. Lightwave Technol., Vol. 25, 2007, 1588-1593.

[5] J. D. Bierlein, D. B. Laubacher, J. B. Brown, C. J. van der Poel, "Balanced phase matching in $\mathrm{KTiOP}_{4}$ waveguides", Appl. Phys. Lett., vol. 56, 1990, 1725-1727.

[6] Z. Weissman, A. Hardy, M. Katz, M. Oron, D Eger, "Second-harmonic generation in Bragg-resonant quasi-phase-matched periodically segmented waveguides", Opt. Lett., Vol 20, No 7, 1995, 674-676

[7] W.P. Risk, S.D. Lau, and M.A. McCord, "Compact Blue/Green Lasers Conference", OSA Technical Digest Series,Vol. 1, paper CWI14, 1994. 
[8] P. Aschieri, V. Doya and A. Picozzi, "Complex behaviour of a ray in a Gaussian index profle periodically segmented waveguide", J. Opt. A : Pure Appl. Opt. 8, 2006, 386-390.

[9] V. Rastogi, A. K. Ghatak, D. B. Ostrowsky, K. Thyagarajan, and M. R. Shenoy, "Ray analysis of parabolic-index segmented planar waveguide", Appl. Opt., vol. 37, No. 21, 1998, pp. 4851-4856.

[10] K. Thyagarajan, V. Mahalakshmi, and, M. R. Shenoy, "Propagation characteristics of planar segmented waveguides with parabolic index segments", Opt. Lett., vol. 19, no. 24, 1994, pp. 2113-2115.

[11] L. W. Casperson, "Beam propagation in periodic quadratic-index waveguides", App. Opt., Vol. 24, No. 24, pp. 4395-4403, (1985)

[12] S.S. Abdullaev, "Chaos and dynamics of ray in waveguide media", edited by G. M. Zaslavky, Gordon and breach science publishers, (1993).

[13] F. Faure, "Propagating modes in periodic wave guide in the semiclassical limit", J. Phys. A: Math. Gen. 35, 1339-1356, (2002).

[14] G. M. Zaslavsky, S. S. Abdullaev, "Chaotic transmission of waves and "cooling" of signals." Chaos.,7, 1997, 182-186.

[15] A. Ghatak and K. Thyagarajan, "Introduction to fiber optics", Cambridge University Press,1998.

[16] M. De Micheli, J. Botineau, S. Neveu, P. Sibillot, D. B. Ostrowsky , "Independent control of index and profile in proton-exchanged lithium niobate guide", Opt. Lett., vol. 8, 1983, 114-115. 
[17] A Java applet simulation of the ray propagation in a gaussian index PSW and the corresponding Poincaré section is avalable at the following adress : http://www.unice.fr/optics/ray_psw.html

[18] V. I. Arnold, "Mathematical Methods of classical mechanics", SpringerVerlag, 1978.

[19] L. Li and J.J. Burke, "Linear propagation characteristics of periodically segmented waveguides", Opt. Lett., vol. 17, pp. 1195-1197, (1992). 


\section{Figure captions}

Fig. 1 : Schematic of the investigated longitudinally periodic waveguide with a period $\Lambda$ and the transverse gaussian index profile of the high index segments. Everywhere else, the index is constant and is equal to the substrate index $n_{2}$.

Fig. 2 : Typical ray path in a transverse gaussian index profile PSW. If $\theta_{i} \neq 0^{\circ}$ or $x_{i} \neq 0$, the ray path oscillate with a period $Z_{p}$ which depends on initial conditions whereas the ray path is a straight line if $\theta_{i}=0^{\circ}$ and $x_{i}=0$.

Fig. 3 : Poincaré section of a PSW with the following parameters : $D C=0.5, \Lambda=50.7 \mu \mathrm{m}, \delta n=0.029, w=3.5 \mu \mathrm{m}$ (a) and the corresponding dispersion map for the same waveguide (b).

Fig. 4 : Dispersion curve as a function of the position of the input ray for $\theta=0^{\circ}$ for a PSW (continuous line) and for the equivalent continuous waveguide (doted line).

Fig. 5 : Dispersion values as a function of the index difference $\delta_{n}$ for different periods for a given input condition $\left(\theta_{i}=0^{\circ}\right.$ and $\left.x_{i}=1.5 \mu \mathrm{m}\right)$, others PSW parameters are $D C=0.5, w=5 \mu \mathrm{m}$.

Fig. 6 : Schematic of the investigated longitudinally periodic waveguide with an asymmetric gaussian index profile, $w_{1} \neq w_{2}$. Outside of the high index segments, the index is constant and equal to the substrate index $n_{2}$.

Fig. 7 : Poincaré section of a PSW with the following parameters : $D C=0.5, \Lambda=18 \mu \mathrm{m}, \delta n=0.02, w_{2}=10 \times w_{1}=4.3 \mu \mathrm{m}$ (a) and the corresponding dispersion map (b).

Fig. 8 : Dispersion curve as a function of the position of the input ray for $\theta=0^{\circ}$ for a PSW (continuous line) and for the equivalent continuous 
waveguide (doted line).

Fig. 9 : Dispersion values as a function of the index difference $\delta_{n}$ for two different periods for a given input condition $\left(\theta_{i}=0^{\circ}\right.$ and $\left.x_{i}=0.5 \mu \mathrm{m}\right)$, others PSW parameters are $D C=0.5, w_{2}=6 \times w_{1}=5 \mu \mathrm{m}$ 


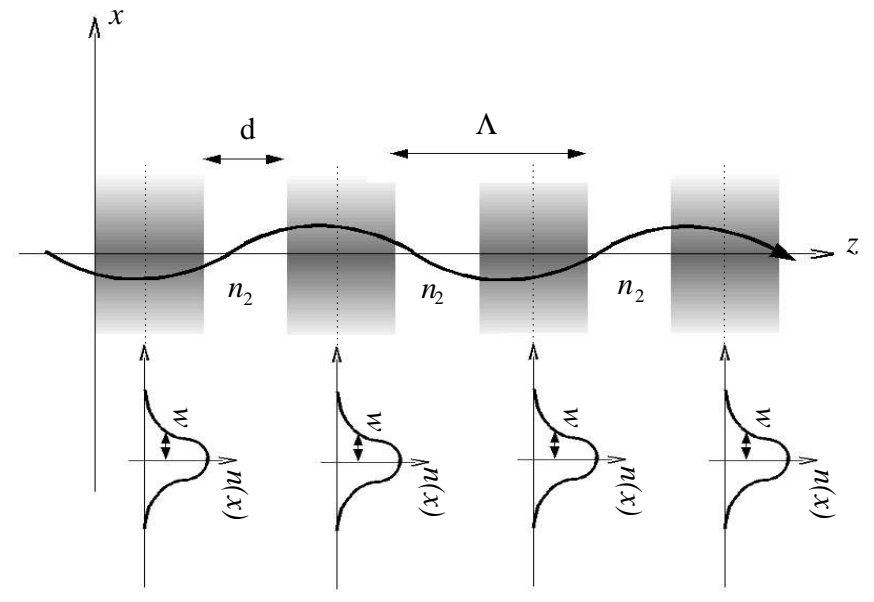

Figure 1: 


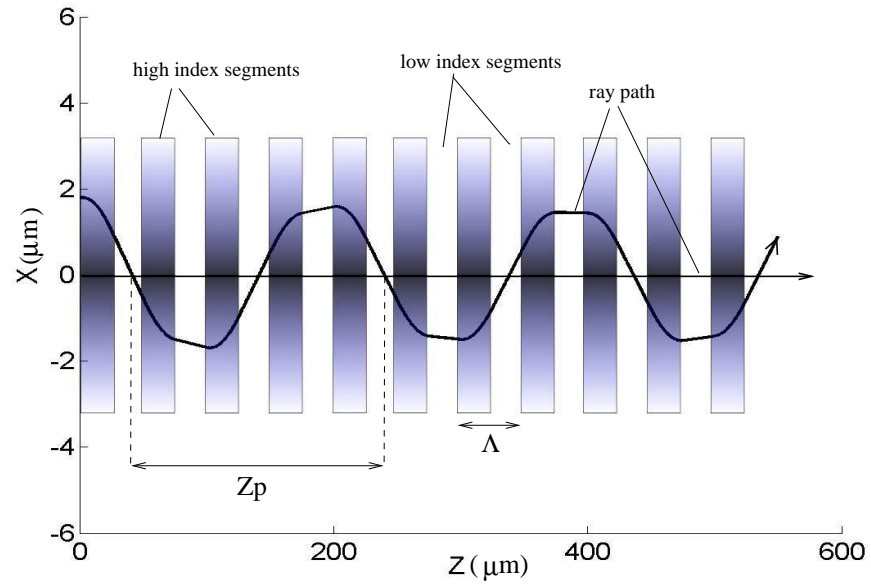

Figure 2: 


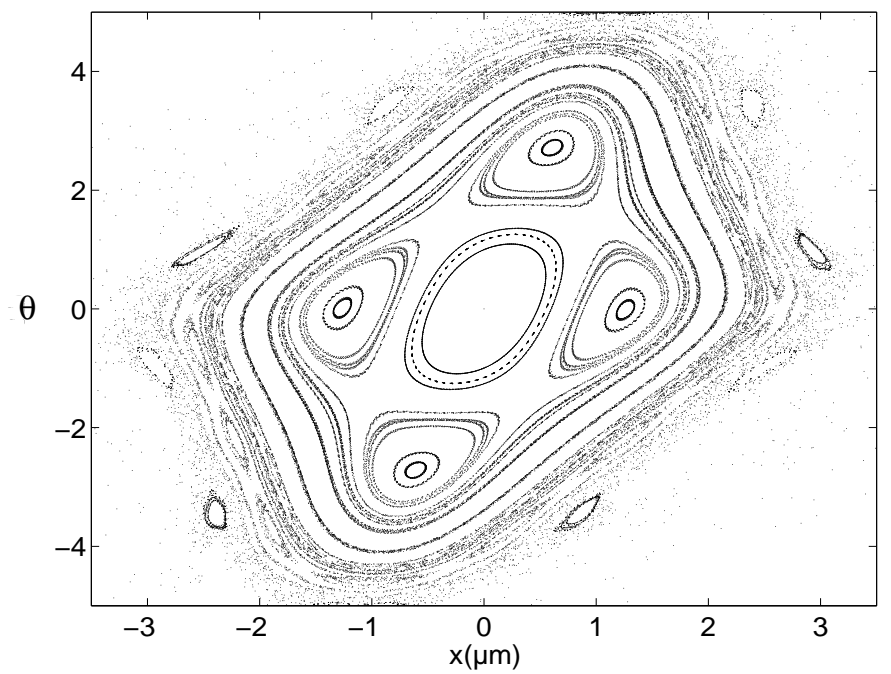

(a)

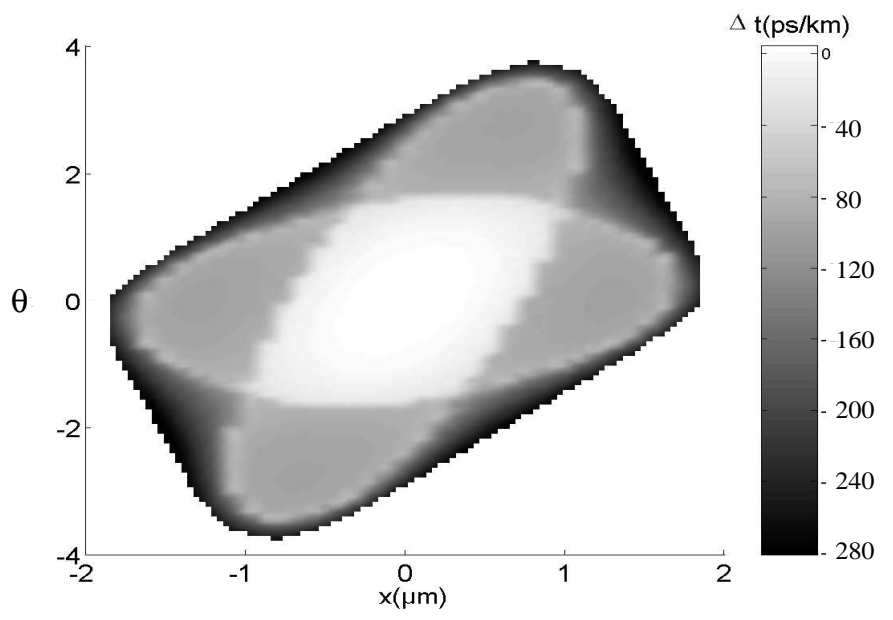

(b)

Figure 3: 


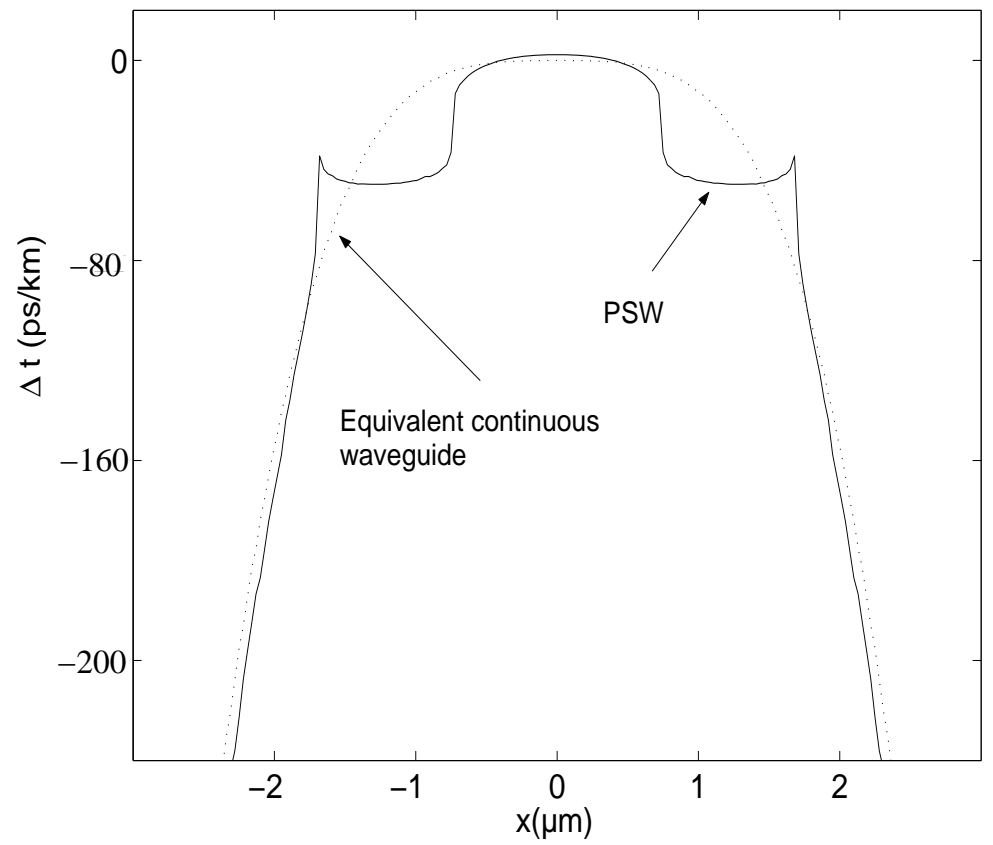

Figure 4: 


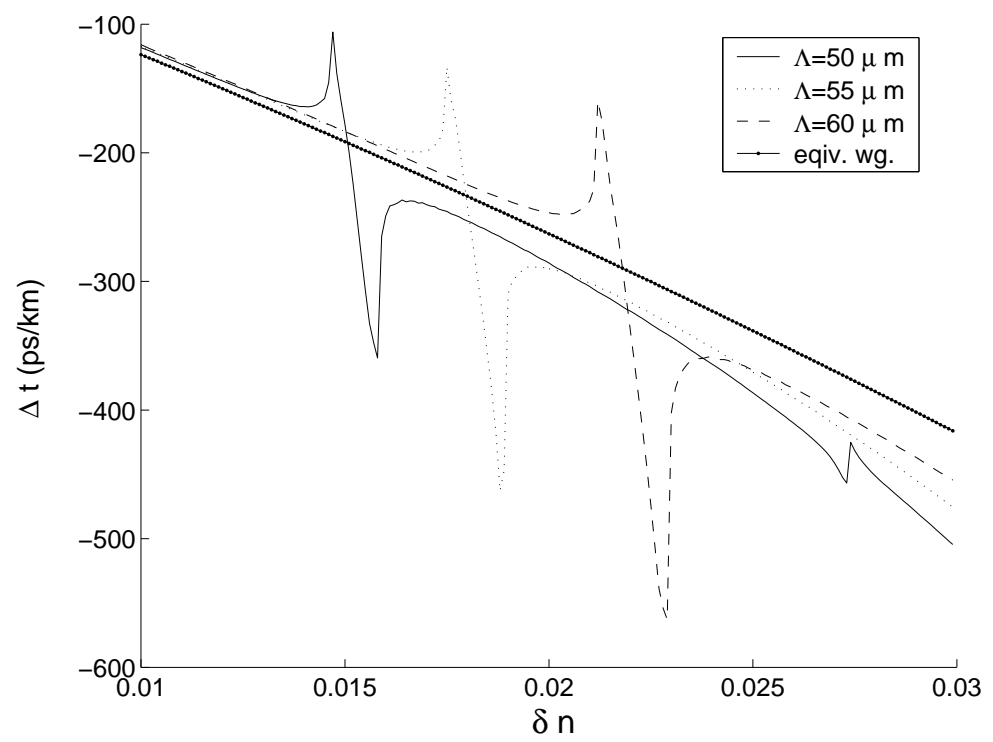

Figure 5: 

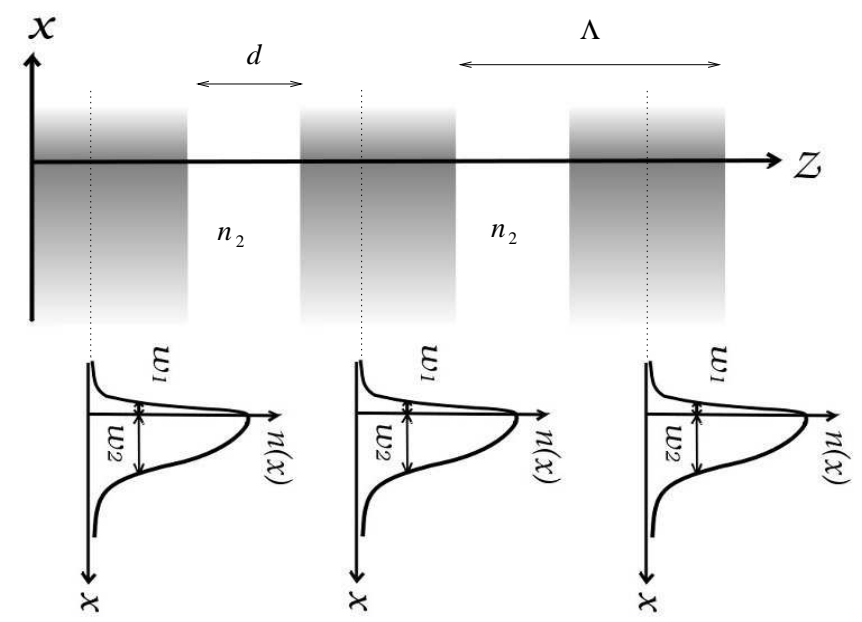

Figure 6: 


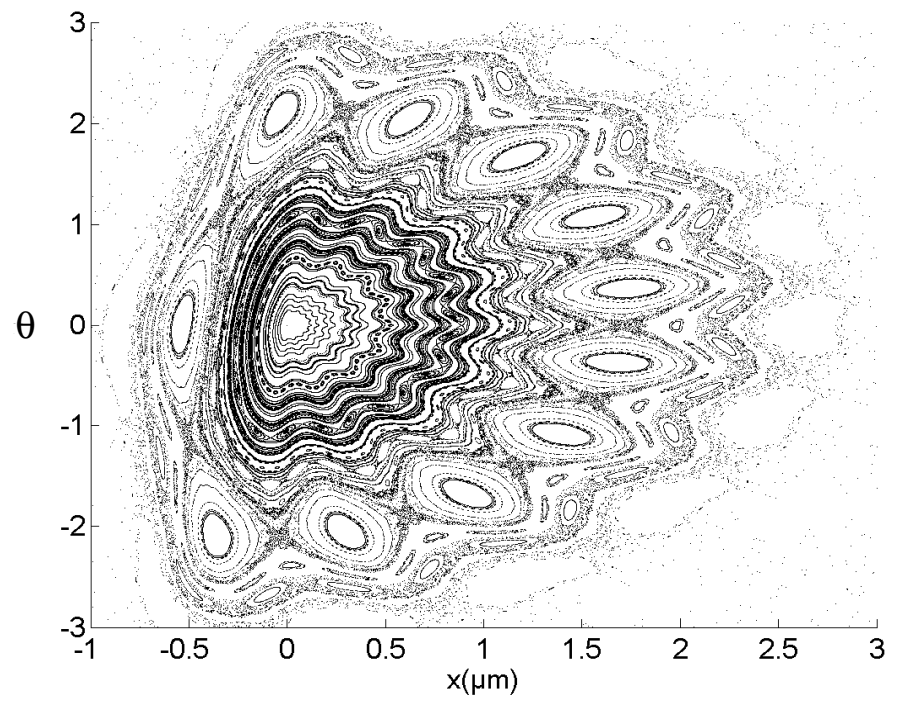

(a)

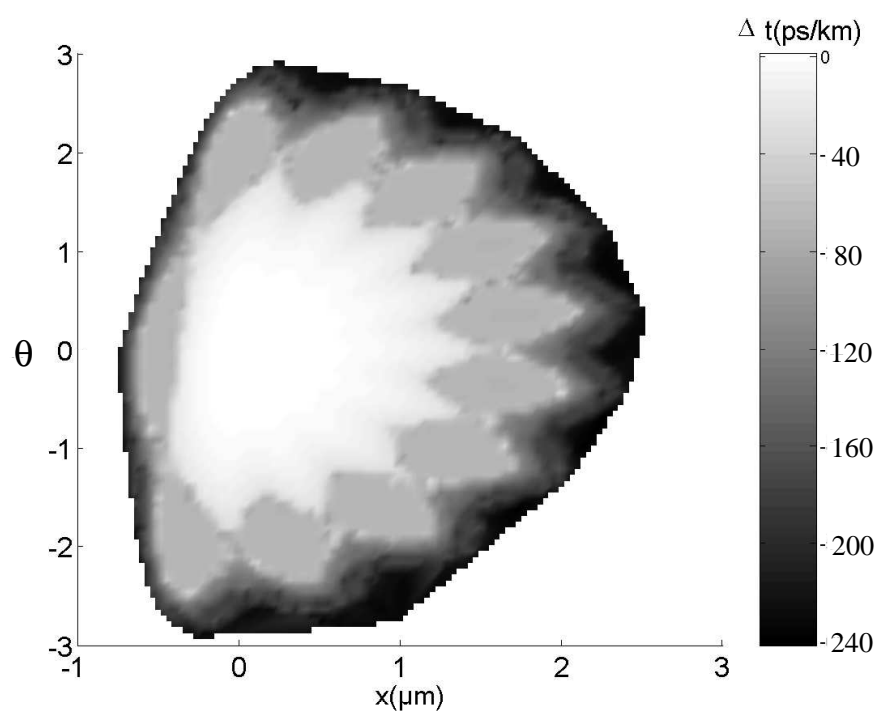

(b)

Figure 7: 


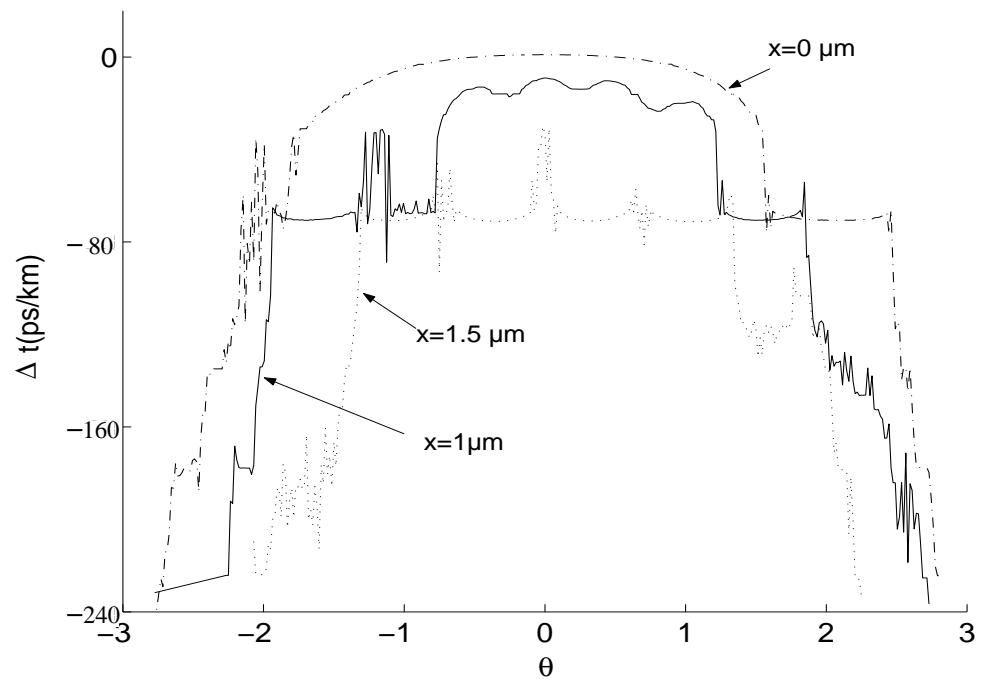

Figure 8: 


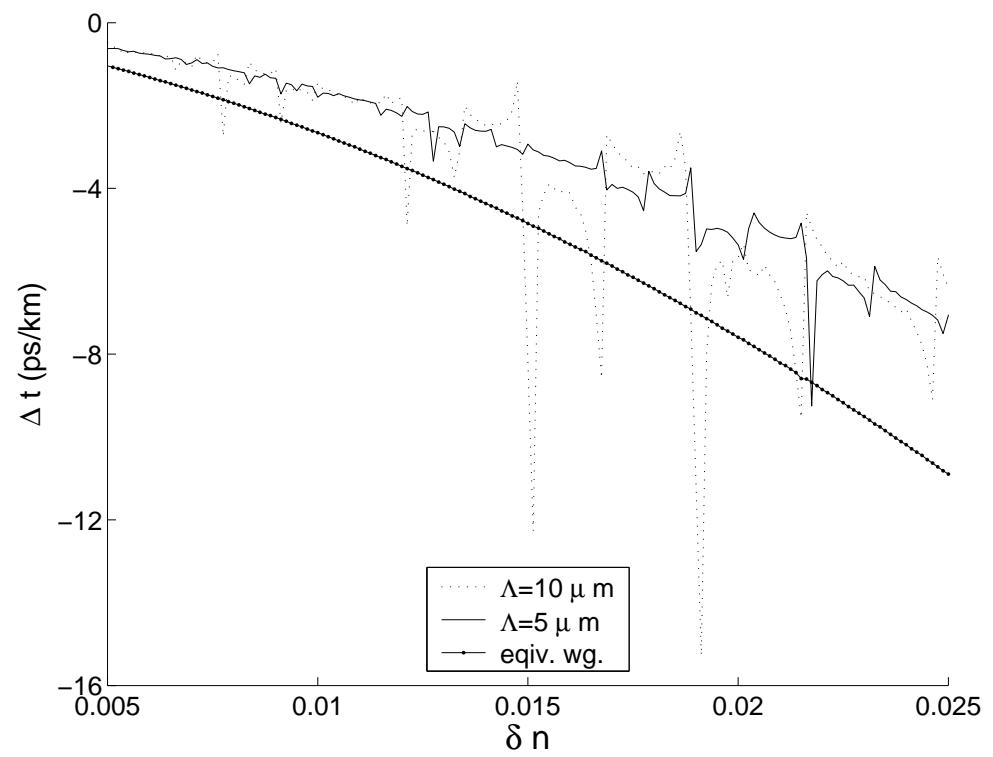

Figure 9: 